ENCYCLOPÉDIE Encyclopédie berbère

BERBERE

26 | 2004

26 | Judaïsme - Kabylie

Kabylie : La langue

Présentation générale

S. Chaker

(2) OpenEdition

Journals

Édition électronique

URL : http://journals.openedition.org/encyclopedieberbere/1431

DOI : 10.4000/encyclopedieberbere.1431

ISSN : 2262-7197

Éditeur

Peeters Publishers

Édition imprimée

Date de publication : 1 mai 2004

Pagination : 4055-4066

ISBN : 2-7449-0452-X

ISSN : $1015-7344$

Référence électronique

S. Chaker, « Kabylie : La langue », Encyclopédie berbère [En ligne], 26 | 2004, document K15, mis en ligne le 01 juin 2011, consulté le 14 décembre 2020. URL : http://journals.openedition.org/ encyclopedieberbere/1431 ; DOI : https://doi.org/10.4000/encyclopedieberbere.1431

Ce document a été généré automatiquement le 14 décembre 2020.

(c) Tous droits réservés 


\title{
Kabylie : La langue
}

\author{
Présentation générale
}

\section{S. Chaker}

1 Le berbère parlé en kabyle est, avec le touareg* et le tachelhit* du Sud-Ouest marocain, une des variétés régionales les plus étudiées et les mieux connues. C'est aussi celle qui a bénéficié du processus de promotion et d'aménagement linguistique le plus précoce et le plus avancé.

2 Les données historiques et sociales expliquent aisément cette situation particulière ; principale région berbérophone, bastion d'une forte et longue résistance à la pénétration française au $\mathrm{XIX}^{\mathrm{e}}$ siècle, située à moins d'une centaine de kilomètres de la capitale Alger, la Kabylie a très tôt attiré les descripteurs français : explorateurs et voyageurs, militaires, missionnaires et scientifiques. Le kabyle a donc fait l'objet d'une attention précise et précoce comme en témoigne la publication dès $1844 \mathrm{du}$ premier dictionnaire de cette langue. Entre 1858 et 1873, le Général Hanoteau, véritable encyclopédiste de la Kabylie, publie à lui seul : sa Grammaire kabyle (1858), ses Poésies populaires du Jurjura (1867) et son œuvre monumentale en trois volumes, La Kabylie et les coutumes kabyles (1873). L'impulsion donnée lors de ces premières décennies de la présence française en Algérie sera décisive et sera rapidement relayée par de nouveaux types d'acteurs, non moins productifs :

- Les religieux chrétiens (principalement de l'ordre des missionnaires d'Afrique, les « Pères blancs » et « Sœurs blanches », fondé par le cardinal Lavigerie en 1868/9), dont le travail de description de la langue berbère de Kabylie se poursuivra sur place sans interruption jusqu'au milieu des années 1970; il donnera le jour notamment à la précieuse série du Fichier de Documentation Berbère (1946-1977) et au Dictionnaire kabyle-français de Jean-Marie Dallet (1982).

- À partir des années 1880, les spécialistes français de l'Université d'Alger qui avec René Basset, qui fut Doyen de la Faculté des Lettres, s'imposera vite comme le principal pôle des études berbères jusqu'à la décolonisation. La Kabylie étant toute proche, l'enseignement du berbère à la Faculté des Lettres d'Alger et à l'Ecole normale de Bouzaréah, de même que les recherches et publications des titulaires de la chaire de berbère (René et André Basset, puis André Picard) porteront principalement sur le 
kabyle ;

- Enfin, dans les dernières décennies du XIX siècle, les berbérisants autochtones, quasiment tous kabyles, émergeront de différents corps de l'appareil administratif français : interprètes-traducteurs militaires et civils (Ben Khouas, Cid Kaoui...), instituteurs (dont le plus connu et le plus fécond fut Boulifa); puis, à partir des années 1930, hommes de lettres (Amrouche, Feraoun, Mammeri*...).

3 Malgré le coup d'arrêt qu'a représenté la suppression de la chaire de berbère de la Faculté des Lettres d'Alger en 1962 et la mise en place d'une politique linguistique et culturelle très anti-berbère dans l'Algérie indépendante, ce capital scientifique et ce potentiel humain constitués pendant la période française n'allaient pas rester sans retombées et sans suites : à partir des années 1960, de nombreux jeunes Kabyles, en Algérie et en France, sensibilisés par le travail des générations antérieures et les prises de positions de quelques rares références en activité (principalement l'écrivain Mouloud Mammeri), se mettront à l'étude de leur langue, soit dans le cadre académique, soit dans des cadres associatifs et militants. Ce double engagement débouchera progressivement sur une véritable renaissance des travaux et publications consacrés au kabyle : études descriptives dans le cadre des différents courants de la linguistique moderne (Chaker, Mettouchi, Naït-Zerrad, Allaoua et tant d'autres) ; études à orientation plus appliquée, dans le domaine de l'aménagement linguistique : notation usuelle, didactique, terminologie (Achab, Naït-Zerrad...).

4 À partir de 1990, l'assouplissement de la position des autorités algériennes vis-à-vis du berbère, avec la création des Départements de Langue et Culture Amazigh à l'Université de Tizi-Ouzou (1990), puis de Bougie (1991), va permettre l'arrivée d'une nouvelle génération, bien plus nombreuse, de jeunes chercheurs berbérisants travaillant en Kabylie même. En une décennie, les travaux de cette nouvelle berbérologie kabyle ont significativement renouvelé la connaissance que l'on pouvait avoir du kabyle, notamment de sa diversité interne ( $C$. Notice suivante « Kabylie : Dialectologie » par K. Naït-Zerrad).

\section{Quelques données sociolinguistiques}

5 La variété kabyle du berbère est la langue maternelle et usuelle de l'immense majorité de la population de Kabylie : près de $85 \%$ des habitants de l'ancien département de Tizi-Ouzou ("Grande Kabylie ») se déclare berbérophones natifs (recensement ${ }^{1}$ officiel algérien de 1966). Il convient à ce propos de souligner que les nombreux découpages et redécoupages administratifs de l'entité géolinguistique kabyle opérés par l'Etat algérien ont eu pour conséquence de fragmenter l'aire de la kabylophonie sur au moins cinq départements (wilayat). Tant et si bien que seules les départements de Tizi-Ouzou et de Bougie peuvent être considérés comme presque entièrement berbérophones; les autres fragments de l'aire kabyle sont intégrés dans des unités administratives périphériques, dont la plus grande partie est arabophone (Sétif, Bouira, Boumerdes). Ce démembrement administratif de la Kabylie historique et culturelle ne facilite évidemment pas l'évaluation démographique de la berbérophonie dans la région.

On peut néanmoins estimer, sur la base de la projection des chiffres connus, la population kabylophone à environ 5,5 millions de personnes, dont 3 à 3,5 millions vivent en Kabylie même et 2 à 2,5 million constituent la diaspora, dans les grandes 
villes d'Algérie (surtout Alger), mais aussi en France où vivent probablement près d'un million de Kabyles.

7 En Kabylie, l'usage du berbère est tout à fait prédominant ; langue d'usage général dans les échanges quotidiens, villageois et urbains et pour toutes les générations, le berbère n'est pas même vraiment concurrencé dans les espaces officiels accessibles au public (administrations municipales, postes etc.); les seuls lieux de Kabylie où l'on peut constater une présence de l'arabe classique sont les espaces institutionnels formels, placés sous le contrôle direct de l'administration centrale de l'Etat: Ecoles, tribunaux, gendarmeries... Bien sûr, dans les zones de contact entre populations arabophones et berbérophones, le bilinguisme berbère/arabe dialectal est de règle; mais il n'est pas toujours unilatéral : dans de nombreux cas, les arabophones apprennent et utilisent le berbère. Les deux capitales de la Kabylie, Bougie et Tizi-Ouzou, illustrent bien cette pression du berbère : dans les deux cités, le noyau historique ancien de la population était arabophone ; l'exode rural massif de l'après-indépendance, a changé totalement le peuplement de ces deux villes et a généralisé l'usage du berbère.

8 En fait, en Kabylie, notamment dans les couches moyennes scolarisées, c'est plutôt le français qui concurrence significativement le berbère, bien sûr à l'écrit, mais aussi dans toutes les situations formelles ou requièrant une certaine élaboration linguistique (usages techniques et scientifiques, politiques...). Cette tendance est confirmée par de nombreux indices objectifs: prégnance de la presse francophone en Kabylie (avec existence de plusieurs titres régionaux), prégnance des chaînes de télévision françaises, multiplication des écoles privées francophones, usage commercial et publicitaire quasi exclusif du français... L'évolution sur ce plan est tout à fait impressionnante depuis le début des années 1990 : dans les espaces publics - en-dehors des sites officiels de l'Etat central -la langue arabe à totalement disparu au profit du berbère (en notation tifinagh et latine) et du français.

\section{Quelques traits linguistiques marquants du kabyle}

9 On rappellera en premier lieu que les variétés régionales actuelles du berbère (les «dialectes ») résultent du processus historique de longue durée d'arabisation d'une partie du territoire de l'Afrique du Nord; la conséquence mécanique de cette réalité, soulignée dans la notice «Dialecte» (EBXV, 1995), est que les "géo-lectes» contemporains ne présentent pas nécessairement une profonde unité linguistique : ils résultent de l'assemblage « par soustraction » de parlers qui, originellement, pouvaient appartenir à des aires dialectales distinctes. Tel semble bien être le cas de la Kabylie, comme tend à le montrer la notice suivante « Kabylie : Dialectologie ».

Néanmoins, il existe une image, et sans doute une forme, dominante du kabyle, fondées à la fois sur le poids démographique, l'extension géographique, la représentation dans les études berbères et la bibliographie; la représentation aussi dans la production culturelle moderne - que ce soit la chanson ou l'écrit littéraire. Il s'agit de la variété de kabyle parlée dans ce qu'il est convenu d'appeler la "Grande Kabylie» et principalement la Kabylie du Djurdjura ou Haute Kabylie, le kabyle des «Zouaouas », comme l'on disait autrefois. Le nombre de travaux consacrés à cette région depuis le $\mathrm{XIX}^{\mathrm{e}}$ siècle, mais aussi le poids considérable qu'ont joué les élites locales dans l'étude et la valorisation du berbère - de Boulifa à Mammeri - expliquent largement la prédominance et la visibilité de cette forme de kabyle. Les limites géographiques de ce 
«kabyle classique » sont assez floues et il n'est pas sans connaitre de sensibles variations (notamment phonétiques) en son sein. Cependant, on peut en cerner les tendances lourdes.

\section{a) Phonétique et phonologie}

11 Le kabyle, en dehors des franges de la Kabylie orientale, est caractérisé par une série de traits phonétiques, fortement représentés, qui donnent à ce dialecte une «identité phonétique » marquée :

Spirantisation des occlusives

$\mathrm{b}>\underline{\mathrm{b}}([[\beta]) \mathrm{d}>\underline{\mathrm{d}}([\delta]) \mathrm{d}>\underline{\mathrm{d}}([\delta]) \mathrm{g}>\mathrm{g}([\gamma])$

$\mathrm{t}>\underline{\mathrm{t}}([\theta]) \mathrm{k}>\underline{\mathrm{k}}([\mathrm{c}])$

(entre parenthèses : API)

12 La spirantisation - qui est un affaiblissement de l'occlusion - est systématique et concerne toutes les consonnes, sauf dans quelques rares contextes protégés où la consonne est maintenue occlusive; il s'agit donc dans ce cas de variantes contextuellement conditionnées :

Berbère (chleuh) : $\mathrm{t}$ kabyle : [t] țamyart « vieille»

[t] après $/ \mathrm{l} / \mathrm{ou} / \mathrm{n} /$

tamllalt « œuf », ddant « elles sont allées »

Berbère (chleuh) : d kabyle : [ $\underline{d}] \underline{d} a$, « ici »

[d], après $/ \mathrm{l} / \mathrm{ou} / \mathrm{n} /$

anda « où ? » (comparez avec : anid $a)$

aldun « plomb"

Berbère (chleuh) : k kabyle : [k] a $a$ al « terre »

[k], après $/ \mathrm{r} /, / \mathrm{l} /$

rku « pourrir », tankra « se lever », tilkin « poux »

Berbère (chleuh) : $\mathrm{g}$ kabyle : [g] taga « carde »

[g], après $/ \mathrm{r} /, / \mathrm{n} /$

argu « rêver ", ngef « haleter »

On notera qu'en-dehors du kabyle, la spirantisation touche également la plupart des dialectes berbères du Nord, à l'exception du chleuh (où elle est très localisée et limitée) ; mais en kabyle, cette tendance est particulièrement forte et large et concerne toutes les occlusives simples du berbère.

14 Affriction des dentales (surtout $\left[\mathrm{t}^{\mathrm{s}}, \mathrm{tt}^{\mathrm{s}}\right]$, notées habituellement [Ţ, Тฺ̧], et des prépalatales.

15 Les affriquées dentales sourdes $\left[\mathrm{t}^{\mathrm{s}}, \mathrm{t} \mathrm{t}^{\mathrm{s}}\right]$ sont extrêmement fréquentes, notamment dans les parlers de Grande Kabylie. Elles correspondent toujours à un [tt] tendu en chleuh et dans les autres dialectes berbères; elles doivent leur fréquence à leur statut grammatical car $\left[\mathrm{tt}>\mathrm{tt}^{\mathrm{s}}\right]$ est la marque préfixée de l'aoriste intensif de nombreuses catégories de verbe (awi $\rightarrow$ ttawi) et résulte également, de manière quasi obligatoire, de la séquence $/ d+t /$, particulièrement fréquente dans les syntagmes nominaux :

préposition $d$ « avec » + Nom féminin : tamyart $d$ wemyar ( "le vieux et la vieille »), mais :

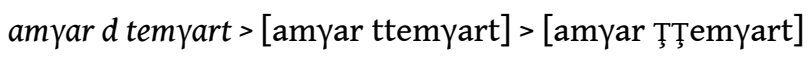

morphème de prédication nominal $d$ + Nominal féminin : $d$ amyar (« c'est un vieux »), mais : $d$ tamyart > [ttamyart] > [ᄁ̧̧̧amץart] (« c'est une vieille »). 
16 En Grande Kabylie, l'extension du phénomène d'affriction varie selon les parlers et les contextes (dans un contexte donné, certains parlers peuvent avoir [tt] au lieu de [tts $]$ ) ; on aura, par exemple :

- [ttamyart] (</d tamyart/), « c'est une vieille/elle est âgée », chez les Aït Yanni, mais : - [ŢŢamyart], chez les Aît Iraten.

17 En Petite Kabylie, à l'Est de Bougie, la dentale affriquée disparaît complètement et l'on a toujours maintien de [tt] (Cf. Notice «Kabylie: Dialectologie » ci-dessous). En conséquence, $\left[\mathrm{tt}^{\mathrm{s}}\right]$ doit être considéré comme une simple variante locale $\mathrm{du} / \mathrm{tt} /$ berbère et n'est pas noté dans la notation phonologique ni dans l'écriture usuelle.

18 En revanche, les affriquées pré-palatales $\left[\mathrm{t}^{\mathrm{s}}\right]$ et $\left[\mathrm{d}^{\check{z}}\right]$ sont plus stables et nettement distinctives en kabyle et sont notées respectivement :

$\left[\mathrm{t}^{\grave{s}}\right]=\check{c}: \check{c} \check{c}$, « manger » (distinct de [ ̌̌š], noté $c c$ (cri pour chasser un animal);

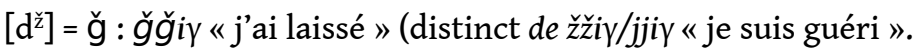

19 La labio-vélarisation : il s'agit d'une co-articulation vocalique furtive $\left([\mathrm{u} / \mathrm{w}]\right.$, notée $\mathrm{C}^{\mathrm{w}}$ ou $\mathrm{C}^{\circ}$, qui accompagne la labiale $\left(\mathrm{bb}^{\circ}\right)$ et les palato-vélaires $\left(\mathrm{k}^{\circ} / \mathrm{kk}^{\circ}, \mathrm{g}^{\circ} / \mathrm{gg}^{\circ}, \mathrm{\gamma}^{\circ}, \mathrm{x}^{\circ}, \mathrm{q}^{\circ} /\right.$ $\left.\mathrm{qq}^{\circ}\right):$ yebb ${ }^{\circ} a$ « il est cuit »; $a k^{\circ} \mathrm{er}$ " voler » : al $\gamma^{\circ} \mathrm{em}$ « chameau »; ameqq ${ }^{\circ}$ ran " grand »...

20 Le phénomène est largement répandu dans tous les dialectes berbères Nord, avec cependant un fréquence et une extension variable selon les parlers. Mais en kabyle :

- la labio-vélarisation est très rarement distinctive et cette pertinence éventuelle est toujours très localisée ;

- au sein des parlers qui connaissent la labio-vélarisation, celle-ci est souvent instable : des parlers voisins ne la placeront pas nécessairement sur les mêmes séquences (ainsi ameqq ${ }^{\circ}$ ran " grand ", chez les Aï Iraten, mais ameqqran chez les Aït Yanni);

- certains parlers de Petite Kabylie (Bougie et toute la côte de Petite Kabylie) ignorent complètement la labio-vélarisation : aker "voler " (au lieu de ak er), ameqqran " grand ", au lieu de ameqq ${ }^{\circ}$ ran, etc.

\section{Vocalisme et accentuation}

Le système vocalique du kabyle est identique à celui de tous les dialectes Nord, très simple, réduit au triangle vocalique de base : /a/, /i/, /u/. Sur ce plan, le seul trait notable, mais qui demande encore de sérieuses vérifications instrumentales, paraît être la fréquence et la consistance de la voyelle neutre [ə] qui, au niveau phonologique, doit être considérée comme un simple « lubrifiant phonique » destiné à éviter les suites de trois consonnes, sans pouvoir distinctif et à l'apparition prédictible. Si, pour ce qui est du statut de [ə], les données kabyles sont celles de tous les dialectes berbères Nord, cette voyelle a en kabyle, contrairement au tachelhit par exemple, une réelle consistance phonétique, notamment une durée souvent très perceptible. Il n'est pas même totalement exclu que l'on puisse trouver, sporadiquement, des cas où ce schwa serait pertinent (par. ex. : sseyr « enseigner » $\neq$ ssyer « faire sécher »?).

Au niveau de l'accent, nos travaux (Chaker 1995b, chap. 8 et 1995c) ont montré que le kabyle à un accent clairement mélodique (ou « musical », fondé sur les modulations de la fréquence fondamentale, $\mathrm{F}^{\circ}$ ), contrairement à ce que pensaient et ont écrit la plupart des berbérisants ${ }^{2}$. Sa position distingue assez systématiquement le nom et le verbe. On ne dispose pas d'études qui permettraient la comparaison avec les autres grands dialectes berbères; il est cependant assez probable que cette nature musicale de l'accent est propre au kabyle, ou en tous cas, plus nettement marquée dans ce dialecte. 
23 En définitive, à l'exception des affriquée pré-palatales /ğ/ et /č/, tous les sons spécifiques du kabyle ne peuvent, du moins dans le cadre d'une phonologie pan-kabyle, être considérés comme des phonèmes, mais uniquement comme des variantes locales, voire micro-locales. Pourtant, au niveau perceptif et subjectif, ils donnent au kabyle une identité forte, par rapport aux autres variétés de berbère et à l'arabe dialectal environnant.

24 La spirantisation généralisée, la fréquence et la consistance de la voyelle neutre et un accent de type mélodique font que le kabyle est généralement perçu comme une langue " chantante ", volontiers comparée à l'anglais par les arabophones.

\section{b) Morpho-syntaxe}

Au plan de la morphologie et de la syntaxe, deux traits principaux peuvent être retenus comme caractéristiques du kabyle :

- La très bonne conservation de la conjugaison à suffixe du thème de prétérit des verbes d'état et de qualité ; cette conjugaison particulière - sans doute un reflet lointain de la conjugaison à suffixe sur base nominale du chamito-sémitique ( $f f$. "Indice de personnes » EBXXIV, 2001, et Chaker 2004) -, a laissé des traces dans de nombreux dialectes berbères (notamment en touareg Ahaggar), mais nulle part elle n'a été aussi bien conservée qu'en kabyle où elle reste non seulement complète, mais usité pour un nombre considérable de verbes, sans doute plus de 150 (Chaker 1983).

- La très grande vitalité de la phrase nominale et de tous les types de prédicats nonverbaux. Non seulement le type classique, attesté dans tous les dialectes méditerranéens, $d$ + Nom (morphème de prédication spécifique + nominal indépendant) est particuliement fréquent, mais on peut y relever un nombre impressionnant d'autres types de prédicats non verbaux, utilisant des outils non-spécifiques: prépositions diverses, adverbes et formes diverses invariables, certains nominaux..., le plus souvent combinés à des affixes personnels des diverses séries pronominales (Chaker 1983). En kabyle, presque tout peut être prédicat, à condition d'être combiné avec la personne grammaticale, configuration très éclairante pour la théorie générale de la prédication et pour la genèse de la catégorie du verbe.

\section{c) Lexique}

26 Comme la plupart des dialectes Nord, le kabyle est avant tout marqué par une très forte influence de l'arabe, classique et dialectal, auxquels il a emprunté une masse considérable de lexèmes; entre 35 à $40 \%$ selon nos estimations faites sur la base d'une liste-diagnostic (Chaker 1984, chap. 11).

Même si ces emprunts à l'arabe obéissent globalement à un déterminisme socioculturel relativement transparent (pression culturelle, technologique et économique d'une langue exerçant sa domination depuis treize siècles), il n'empêche que l'adoption de beaucoup de ces unités ne peut se comprendre que par des raisons internes à la société berbère, notamment par les tabous linguistiques qui induisent une stratégie généralisée d'évitement par le moyen de l'euphémisme* favorisant l'emprunt. Par ailleurs, cette pression de l'arabe n'est pas exclusive :

- de phénomène étonnants de conservatisme lexical pour certaines notions : par. ex., celle d'« écrire/écriture » : aru/tira, alors qu'un dialecte réputé conservateur comme le 
touareg l'a remplacé par l'emprunt arabe ekteb;

- d'une très forte capacité d'intégration phonologique, morphologique et lexicosémantique : des termes comme taktabt/tiktabin « livre(s) » (de l'arabe kitâb) ou tamdint/ timdinin «ville (s) » (de l'arabe (madîna) sont devenus en kabyle de parfaites formes berbères.

Mais l'arabe n'a pas été, loin de là, la seule langue à avoir exercé une influence significative sur le kabyle; le français, depuis la colonisation, joue également un rôle considérable dans la formation du lexique kabyle, sans aucun doute bien au-delà de ce que connaissent tous les autres dialectes berbères. La relation particulière de la Kabylie à la France et à la langue française (émigration très ancienne vers la France, scolarisation précoce, importance des élites francophones...) explique que le kabyle soit truffé d'emprunts au français, le plus souvent "gommés ", par réflexe puriste, dans les collectes lexicographiques. Tous les vocabulaires de spécialités modernes (technologique, scientifique, administratif et politique) sont généralement empruntés au français et font partie de l'usage courant, contrairement au même vocabulaire emprunté à l'arabe classique par les arabisants.

On notera que depuis 1970 environ, avec le développement des initiatives d'aménagement linguistique, un important travail de lutte contre l'envahissement lexical arabe, mais aussi français, a été engagé par recours à moyens divers: revivification de lexèmes anciens tombés en désuétude, emprunts à d'autres dialectes berbères (principalement le touareg), formation de néologismes par dérivation et composition sur des racines berbères (voir ci-dessous). Cette dynamique, par ailleurs tout à fait légitime, confine parfois à une véritable entreprise «d'épuration linguistique » qui n'échappe ni à l'excès, ni au ridicule (sur ce sujet voir l'excellente analyse de Achab 1996).

\section{Aménagement linguistique et développements récents du kabyle}

\section{Le passage à l'écrit}

Le souci de définir et de diffuser une graphie usuelle pour leur langue a été partagé par tous les berbérisants autochtones depuis le début du $\mathrm{xx}^{\mathrm{e}}$ siècle, qu'ils appartiennent à la sensibilité «culturaliste» des instituteurs et hommes de lettres kabyles (Boulifa, Amrouche, Feraoun, Mammeri...) ou à celle des militants "politiques" indépendantistes (Aït-Amrane, Aïche... Cf. Chaker 1998, chap. 2 et 5). Formés à l'Ecole française, ayant acquis leurs instruments d'analyse à partir de la langue française, tous ces acteurs sociaux diffusent, depuis un siècle, des graphies du berbère à base latine.

La volonté de sortir la langue de la stricte oralité se traduit par la publication d'importants corpus littéraires ou de textes sur la vie quotidienne. Dans le domaine littéraire, surtout, le support écrit imprimé va suppléer significativement la transmission orale et la mémoire collective. Vers 1945-50, la diffusion de l'écrit à base latine - en-dehors de tout enseignement formalisé en Kabylie - est déjà suffisamment avancée pour que de nombreux membres des élites instruites kabyles soient capables de composer et écrire le texte de chansons, de noter des pièces de poésie traditionnelle. 
transpositions directes des habitudes orthographiques françaises avec notamment de
nombreux digrammes ( $\mathrm{ch}$ », « ou », «th », «dh »), une grande difficulté à distinguer les voyelles $(/ \mathrm{i}, \mathrm{u} / \mathrm{)}$ des semi-voyelles $(/ \mathrm{y}, \mathrm{w} /)$, une prolifération des notations de la voyelle neutre («schwa»). Avec le temps et les développements de la linguistique berbère à l'Université d'Alger (surtout avec André Basset), les intellectuels locaux subiront l'influence de plus en plus nette des usages scientifiques, largement diffusés en Algérie par une vigoureuse édition berbérisante (jusqu'en 1962). On aboutit dans les années 1960, avec des relais efficaces comme M. Mammeri, à la généralisation de notations latines d'inspiration phonologique, caractérisées par :

- La disparition des digrammes,

- La réduction du nombre de notations de la voyelle «neutre",

- L'élimination de la plupart des caractéristiques phonétiques locales infraphonémiques ou, à tout le moins, leur allégement graphique par le recours à de simples diacrités,

- Une segmentation issue directement de l'analyse syntaxique, avec une forte tendance à l'explicitation des amalgames phoniques, très nombreux en berbère à la jonction de morphèmes.

Reçues par une population ayant assez largement bénéficié d'une scolarisation en langue française, ces notations à base latine se sont solidement implantées en Kabylie. Et c'est dans ces graphies qu'ont été produits au cours des dernières décennies tous les ouvrages « grand public » édités en milieux kabyles.

\section{Une tentative emblématique : la réactualisation des tifinagh}

Insérés dans une aire de vieille culture scripturaire, les Berbères ont depuis toujours vu leur langue et leur culture dévalorisées par leur statut d'oralité. Situation qui a induit dans la période contemporaine une réaction très volontariste visant à démontrer que «le berbère ça s'écrit!». C'est ainsi que l'on peut expliquer l'existence dans la sensibilité berbère d'un fort courant qui prône le retour au vieil alphabet berbère (les tifinagh), qui présente le double avantage de marquer l'appartenance historique incontestable de la langue berbère au monde de l'écriture et d'assurer la discrimination maximale par rapport aux cultures environnantes puisque cet alphabet est absolument 
spécifique aux Berbères. En exhumant cette antique écriture - sortie partout de l'usage depuis des siècles, sauf chez les Touaregs - ces militants se donnent une arme particulièrement efficace dans un environnement où l'écriture est mythifiée, voire sacralisée. Et comme cet alphabet berbère est attesté depuis la protohistoire (au moins le $\mathrm{VI}^{\mathrm{e}}$ siècle avant $\mathrm{J}$-C), les Berbères accèdent ainsi à l'Histoire et à la Civilisation, antérieurement à la plupart des peuples qui ont dominé le Maghreb, notamment les Arabes! Les tifinagh permettent aux Berbères de ne plus être catalogués parmi les Barbares et autres primitifs, pour qui la seule alternative est de se fondre dans les « grandes » cultures (écrites), en l'occurrence la culture arabo-islamique...

Ce sont certainement ces deux facteurs (historicité et spécificité) qui fondent l'engouement pour les tifinagh non seulement en Kabylie, mais aussi dans toutes les autres régions berbérophones (Maroc, domaine touareg), surtout dans les milieux militants et populaires. Le groupe (« l'Académie Berbère » de Paris) qui initie, au début des années 1970, les néo-tifinagh était d'ailleurs une organisation qui recrutait essentiellement des prolétaires et des étudiants directement issus de la Kabylie, ayant encore toutes leurs attaches dans le monde rural.

Le linguiste peut émettre les plus extrêmes réserves, théoriques et pratiques, sur ces tentatives de réintroduction des tifinagh. Il pourra même montrer que cette option a été conduite sur des bases linguistiquement aberrantes puisqu'il s'agit en fait d'une notation phonétique du kabyle à base tifinagh! La solution préconisée est donc doublement anti-économique : au plan graphique et au plan du principe sous-jacent à la notation. Mais les arguments du théoricien semblent de peu de poids face à la volonté de matérialiser, à travers une écriture spécifique, une identité distincte. C'est ce qui permet de comprendre l'envahissement de l'espace public en Kabylie par cette écriture, y compris au niveau de la signalétique officielle municipale. Il est d'ailleurs amusant de constater que plus de 30 années après leur mise en circulation par des militants radicaux kabyles, l'institution marocaine adopte ces «néo-tifinagh » comme alphabet officiel du berbère (décision de l'Institut Royal pour la Culture Amazigh) : les précurseurs de l'Académie berbère de Paris n'espéraient certainement pas un tel succès!

\section{La néologie}

La volonté d'insérer la langue berbère dans le monde moderne et de l'utiliser à des fins de communication autres que villageoises et traditionnelles a également très tôt induit une dynamique de création lexicale parmi les militants et intellectuels kabyles.

Les «berbéro-nationalistes", militants politiques de la branche radicale du nationalisme algérien, sont les premiers à s'être engagés, dans les années 1940, dans la voie de l'innovation lexicale : ce sont eux qui, les premiers, sont allés puiser dans les ressources des autres dialectes berbères (touareg, Maroc, Mzab...) pour enrichir et moderniser le vocabulaire kabyle. Nombre de néologismes socio-politiques introduits à cette époque en kabyle sont des emprunts à d'autres dialectes berbères.

Depuis 1970, ce travail de création lexicale s'est nettement accéléré, notamment grâce à l'action de personnalités comme Mouloud Mammeri et à quelques groupes associatifs (à Paris) ou informels (Tizi-Ouzou). Terminologie générale «moderne» (Amawal, 1980), lexiques spécialisés (mathématiques, architecture, linguistique, informatique...) ont été mis en circulation au cours des 25 dernières années. Deux voies principales sont 
empruntées pour cette production néologique :

- L'exploitation des potentialités de la dérivation verbo-nominale, très souple, mais nettement sous-utilisée dans les dialectes berbères autres que le touareg. On crée à partir de racines connues tous les dérivés verbaux et nominaux autorisés par le système : ainsi de afeg « voler », on tire asafag, " avion », amsafag « aviateur », tamsafga " aviation », anafag « aérodrome »...

- L'emprunt aux autres dialectes berbères, où l'on va puiser les éléments de vocabulaire « manquants » : tilelli « liberté » vient du touareg, aylan, « nation » du mozabite...

Le caractère pan-berbère de la néologie kabyle s'est fortement accentué au cours de la période récente : le touareg, secondairement le chleuh, sont massivement utilisés dans toutes les entreprises néologiques.

\section{La question du statut}

Après trois décennies d'ostracisme vis-à-vis du berbère et de politique linguistique d'arabisation (Cf. Chaker 1989/98), l'Etat algérien a sensiblement assoupli sa position à l'égard de la langue et de la culture berbères. Le berbère a retrouvé sa place en 1990 et 1991 dans l'Université ; depuis 1995, des expériences d'enseignement facultatif de la langue sont menées dans les collèges et lycées. Et, depuis mai 2002, le berbère a même acquis statut de seconde "langue nationale» dans la constitution algérienne. Cette évolution politique et juridique concerne bien sûr l'ensemble de la berbérophone algérienne ; mais elle a eu un impact et une signification toutes particulières en Kabylie dans la mesure où cette région était, depuis plusieurs décennies, à la pointe de la revendication en faveur de langue berbère ( $C f$. Chaker 1989/98).

Ainsi, dès le début des années 1990, un tissu associatif dense s'est mis en place légalement en Kabylie; il a pu élargir et renforcer le travail de promotion et de valorisation de la langue berbère, à travers son enseignement et la diffusion de son écrit; il a aussi significativement contribué à l'aménagement de la langue par la diffusion de la notation usuelle et des néologismes, à travers son rôle dans l'édition littéraire et la presse.

De même, l'enseignement du berbère dans les établissements publics à partir de 1995, assuré essentiellement par des enseignants qui étaient parallèlement des acteurs et militants du monde associatif, a consolidé la position du berbère et la diffusion de son écrit en Kabylie; il est d'ailleurs à noter que ces expériences d'enseignement, initialement programmées pour l'ensemble de l'Algérie, se sont très vite limitées, pour l'essentiel, au seul territoire de la Kabylie, parce qu'elles y correspondaient à une attente sociale, parce que la militance associative locale a pu s'y investir. Actuellement, l'écrasante majorité 3 des élèves qui reçoivent cet enseignement se situe en Kabylie.

\section{Conclusion}

La langue berbère de Kabylie, héritière d'une histoire tourmentée et soumise à de très fortes pressions externes, manifeste désormais une vitalité et un dynamisme certains ; elle est la seule variété du berbère qui ait fait l'objet, depuis plus d'un siècle, d'une prise en charge par ses propres locuteurs; de ce fait, le kabyle est en passe réussir son " passage à l'écrit ", à travers la stabilisation d'une notation usuelle à base latine, par le 
développement d'une littérature écrite déjà conséquente et l'émergence d'usages écrits fonctionnels diversifiés, notamment un usage de presse.

Parallèlement, à la faveur de l'assouplissement de la politique linguistique de l'Etat algérien à partir de 1990, l'engagement massif des Kabyles en faveur de leur langue, a permis de faire du kabyle non seulement une langue enseignée, mais aussi une langue d'enseignement.

Une véritable norme instituée du berbère est donc en cours de constitution en Kabylie, dynamique qui, de fait et contre les aspirations mêmes de ses promoteurs Kabyles, devrait rapidement donner au kabyle le statut de langue particulière dans l'ensemble berbère. Le travail sur la langue menée au cours $\mathrm{du} \mathrm{xx}^{\mathrm{e}}$ siècle rejoindrait alors celui des précurseurs comme Boulifa qui n'hésitaient pas à rédiger, en 1897/1913, une Méthode de langue kabyle.

\section{BIBLIOGRAPHIE}

\section{ORIENTATION BIBLIOGRAPHIQUE (notices K15 et K16)}

La bibliographie sur le kabyle est immense ; on en trouvera un signalement systématique dans les (nombreux) outils bibliographiques berbérisants disponibles : Applegate 1970, Basset 1952, Annuaire de l'Afrique du Nord $1965 \rightarrow$ (Galand, Chaker, Brenier-Estrine), Bougchiche 1977... On pourra aussi se reporter sur Internet au site du Centre de Recherche Berbère (serveur de I'Inalco : $h t t p / / w w w . i n a l c o . f r)$. On ne signale ici que les travaux cités dans le texte et les ouvrages de référence.

АСНАВ R., 1996, La néologie lexicale berbère (1945-1995), Paris/Louvain : Editions Peeters, 1996.

ACHAB R., 1998, Langue berbère. Introduction à la notation usuelle en caractères latins, Paris : Editions Hoggar.

ALlaOUA A., 1988, Un parler berbère d'Algérie (Kabylie : Ayt Ziyan), Thèse de doctorat, Université de Copenhague.

Amawal (lexique), 1980, Paris, Imedyazen. [terminologie technique et moderne berbère-français/ français-berbère]

BASSET A., 1929, Études de géographie linguistique en Kabylie (I. Sur quelques termes concernant le corps humain.), Leroux, Paris.

BASSET A., 1936/1939, Atlas linguistique des parlers berbères. Algérie du nord, 2 voL + cartes, Alger.

BASSET A., 1952 (1969), La langue berbère, Londres.

Basset A., 1957, Articles de dialectologie berbère, Paris, Klincksieck.

BASSET A., picard A., 1948, Eléments de grammaire berbère (Kabylie-Irjen), Algerie, Alger, La TypoLitho.

BEN SEDIRA B., 1887, Cours de langue kabyle, Alger, Jourdan. 
BOULIFA S.A., 1897, Méthode de langue kabyle, cours de première année, Alger, Jourdan.

BOULIFA S.A., 1904, Recueil de poésies kabyles, Alger (Réédition avec présentation par T. Yacine, Paris/Alger, Awal, 1990).

BoULIFA S.A., 1913, Méthode de langue kabyle, cours de deuxième année, Alger, Jourdan.

CHAKER S., 1981, « Dérivés de manière en berbère (kabyle) », Glecs, XVII, 1972-1973 [1981], p. 81-96.

CHAKER S., 1983, Un parler berbère d'Algérie (Kabylie) : syntaxe, Aix-en-Provence : Université de Provence.

CHAKER S., 1984, Textes en linguistique berbère (introduction au domaine berbère), Paris, Cnrs.

CHAKER S., 1989/1998, Berbères Aujourd'hui, Paris, L'Harmattan.

CHAKER S., 1995a, « Dialecte », Encyclopédie berbère XV, p. 2291-2295, Edisud, Aix-en-Provence.

CHAKER S., 1995b, Linguistique berbère. Etudes de syntaxe et de diachronie, Peeters, Paris/Louvain.

CHAKER S., 1995c, « Données exploratoires en prosodie berbère : I. L'accent en kabyle ; II.

Intonation et syntaxe en kabyle », GLECS, 31, 1995, p. 27-54 \& p. 55-82.

CHAKER S., 1997, « Quelques faits de grammaticalisation dans le système verbal berbère », Mémoires de la Société de Linguistique de Paris, V, p. 103-121, Klincksieck, Paris.

CHAKER S., 2001, « Indices de personnes », Encyclopédie berbère XXIV, p. 3725-3729, Edisud, Aix-enProvence.

CHAKER S., 2004 (à paraître), « Les paradigmes personnels du berbère », Morphogenèse des pronoms personnels dans les langues africaines, Table ronde du LLACAN-CNRS, Paris, 13-14 juin 2002.

CHAKER S. (dir.), 2001, Hommes et femmes de Kabylie I, (Dictionnaire biographique de la Kabylie), Aixen-Provence, Edisud.

Contes merveilleux et Fables, Textes nouveaux dans le parler des At-Abbas, 1976, Le Fichier Périodique, Alger.

CREUSAT, 1873, Essai de dictionnaire français-kabyle (Zouaoua), Alger, Jourdan.

DALLET J.-M., 1953, Le verbe kabyle, lexique partiel du parler des At Mangellat, FDB, Fort-National (Alger)

DALLET J.-M., 1982, Dictionnaire kabyle-français, Parler des At Mangellat, selaf, Paris. Dictionnaire Français-Berbère, Paris, Imprimerie royale, 1844. Fichier de Documentation Berbère (FDB), Fortnational (puis Alger), 1946-1977.

GALAND L., 1985, « La langue berbère existe-t-elle ? », Mélanges linguistiques offerts à Maxime Rodinson, Paris, Geuthner.

GALAND L., 1989, « Les langues berbères », La réforme des langues, IV (Histoire et avenir), Hamburg, H. Buske Verlag.

GENEVOIS H., 1955, « Ayt-Embarek, notes d'enquête linguistique », Fichier de Documentation Berbère, 49.

HANOTEAU A., 1858 (1906), Essai de grammaire kabyle, Alger, Jourdan.

HANOTEAU A., 1867, Poésies populaires du Jurjura, Paris.

HANOTEAU A., 1873, La Kabylie et les coutumes kabyles, Paris, Imprimerie nationale (3 vol. ).

(réédition : Paris, Bouchène, 2003). 
HUYGHE R.P, 1901, Dictionnaire kabyle-français. Paris.

LEBLANC (de P.), 1897, Essai de contes kabyles, Batna.

LOUIS de VINCENNES (Sr.), DALLET J-M., 1960, Initiation à la langue berbère (Kabylie) (grammaire), Alger, FDB.

LOUIS de VINCENNES (Sr.), 1971, Vie et travaux des potières aux At-Smazel du Djurdjura. Éléments d'étude psychologique et linguistique, Fort-National, FDB.

MADOUI Kh., 1995, Contribution à la géographie linguistique de la Petite-Kabylie, Mémoire de magister, Université de Bejaia.

MAMMERI M., 1976, Tajerrumt $n$ tmaziyt (tantala taqbaylit), Maspero, Paris.

MAMMERI M., 1980, Poèmes kabyles anciens, Paris, Maspéro.

MOULIERAS A., 1891, Les fourberies de Si Djeh'a, Oran, Perrier. (Réédition : 1987, Paris, la Boîte à documents).

MOULIERAS A., 1893/8, Légendes et contes merveilleux de la Grande Kabylie, Paris, Leroux (2 vol. ).

(réédition : 1999, Aix-en-Provence, Edisud).

NAÏT-ZERRAD K., 1994, Manuel de conjugaison kabyle (le verbe en berbère), L'Harmattan, Paris.

NAÏT-ZERRAD K., 1995, Grammaire du berbère contemporain, I - Morphologie, ENAG, Alger.

NAÏT-ZERRAD K., 2000, "Auxiliaires temporels en berbère », Proceedings of the 2nd World Congress of African Linguistics, Leipzig 1997, p. 669-678, H. Ekkehard Wolff/Orin D. Gensler (eds.), Rüdiger Köppe Verlag, Köln.

NAÏT-ZERRAD K., 2001a, Grammaire moderne du kabyle, Karthala, Paris.

NAÏT-ZERRAD K., 2001b, « Esquisse d'une classification linguistique des parlers berbères », AlAndalus-Magreb 8-9, p. 391-404, 2000-2001, Universidad de Cádiz.

OLIVIER, 1878, Dictionnaire kabyle-français, Le Puy.

PICARD A., 1960, De quelques faits de stylistique dans le parler des Irjen (Kabylie, Algérie). De la phrase inorganique à la phrase organisée, Alger, La Typo-Litho/J. Carbonnel.

RABHI A., 1995, Description d'un parler amazigh : Ayt-Mhend d'Aokas (Bejaia, Algérie). Morphosyntaxe, Mémoire de Magister, Université de Bejaia.

RAHMANI S., 1933, Notes ethnographiques et sociologiques sur les Beni Mh'amed du cap Aokas et les Beni Amrous, Constantine.

RAHMANI S., 1939, Coutumes kabyles du Cap Aokas, Alger.

VENTURE de PARADIS, 1838, Dictionnaire de la langue berbère expliqué en français et en idiome barbaresque précédé d'une grammaire berbère.

\section{NOTES}

1. Ce recensement, qui a été le seul depuis l'indépendance de l'Algérie à comporter une question sur la langue maternelle, donnait en chiffres arrondis : 850000 habitants pour le département de Tizi-Ouzou (dont $85 \%$ de berbérophones) et 1300000 pour celui de Sétif (qui incluait Bougie), dont $40 \%$ de berbérophones, soit 500000 berbérophones pour la Petite Kabyle. Un total donc de 1300000 berbérophones pour la Kabylie, sur une population globale de 12379000 ; auxquels il 
convient d'ajouter un bon million de personnes pour la diaspora, ce qui fait une population kabylophone totale de 2 à 2,3 millions en 1966.

2. Les berbérisants qui se sont penchés sur la question évoquent généralement un accent d'intensité, plutôt faible (Basset, Willms... ; sur ce point, voir nos travaux).

3. Selon les statistiques officielles, cet enseignement concernait 69159 élèves en 2000, dont 50216 ( = 73 \%) en Kabylie (Tizi-Ouzou, Bejaïa, Bouira, Sétif, Boumerdes). Et l'on peut, sans grand risque d'erreur, considérer que les $27 \%$ restants pour le reste du territoire algérien sont majoritairement des Kabyles de la diaspora. Source : L'enseignement de la langue amazighe, Bilan et perspectives, Etude réalisée par Noura TIGZIRI et Amar NABTI, Alger, HCA, octobre 2000.

INDEX

Mots-clés : Dialectologie, Grammaire, Kabylie, Linguistique, Phonétique, Sociolinguistique 